Angela Osterheider, Melanie Leidecker-Sandmann, Sarah Kohler, Volker Stollorz, Meik Bittkowski, Yannick Milhahn und Markus Lehmkuhl

\title{
26. Spotlight: Expert*innen auf dem Gebiet der SynBio. Eine Recherche unter Anwendung des ExpertExplorers
}

\subsection{Einleitung}

Die Synthetische Biologie (SynBio) ist ein höchst interdisziplinärer Forschungszweig, der nicht als einzelnes Forschungsgebiet beschrieben werden kann (zum Forschungsstand siehe Erb, Kap. 8). Vielmehr vereint er ein Spektrum mehrerer naturwissenschaftlicher Disziplinen, wie z. B. Molekular- und Systembiologie, Genetik, Biochemie, Biotechnologie, Physik, Chemie, Informatik und die Ingenieurswissenschaften (Köhler et al., 2017: 61). Die Vielschichtigkeit des Faches führt dazu, dass selbst Wissenschaftler*innen der SynBio sich nicht vorrangig als solche bezeichnen würden, wie auch die German Association of Synthetic Biology (GASB) ausgeführt hat:

Synthetic Biology in Germany covers a broad range of topics, although not many labs or institutes are specifically dedicated to it. Many researchers would not consider themselves first and foremost SynBiologists, but maybe rather molecular biotechnologists, computational biologists or metabolic engineers. ${ }^{1}$ (GASB, 2020)

Sich einen Überblick über das Forschungsfeld der SynBio zu verschaffen, ist somit kein einfaches Unterfangen. Wenn schon Wissenschaftler*innen, die thematisch betrachtet auf dem Gebiet der SynBio forschen, sich nicht zwangsläufig mit diesem Bereich identifi-

1 „Die synthetische Biologie in Deutschland deckt ein breites Spektrum an Themen ab, auch wenn sich nicht viele Labore oder Institute spezifisch mit ihr beschäftigen. Viele Forschende würden sich in erster Linie nicht als SynBiolog*innen bezeichnen, sondern vielleicht eher als molekulare Biotechnolog*innen, Biochemiker*innen oder Expert*innen im Bereich der Stoffwechselwege “ (eigene Übersetzung). 
zieren, dann werden externe Personen wie interessierte Lai*innen, Politiker*innen (die etwa Wissenschaftler*innen für eine Expert*innenanhörung einladen möchten) oder Journalist*innen (die ein Statement eines/r Wissenschaftler*in benötigen) umso schwerer beurteilen können, wer in diesem Bereich tätig ist bzw. fachliche Expertise aufweist.

An diesem Problem setzt unser Beitrag an und verfolgt zwei Ziele: Zum einen möchten wir die Webapplikation ExpertExplorer ${ }^{2}$ als Methode vorstellen, um fachlich ausgewiesene Expert*innen auf einem Forschungsgebiet anhand ihrer Publikationen zu ermitteln. Zum anderen beschreiben wir unter Anwendung des ExpertExplorers die Forschungslandschaft SynBio, indem wir Wissenschaftler*innen mit Expertise auf dem Gebiet der SynBio recherchieren. Der Fokus der Analyse liegt auf in Deutschland tätigen Expert*innen. Um Forschungsleistungen und die damit verbundene Expertise bzw. Reputation auf nationaler Ebene mit den Entwicklungen weltweit zu vergleichen und ins Verhältnis setzen zu können, wird ergänzend auch ein kurzer Blick auf die internationale Forschungslandschaft geworfen. Abschließend reflektieren wir die Funktionsweise des ExpertExplorers sowie unsere Analyse und ziehen ein Fazit.

\subsection{Definition wissenschaftlicher Expertise}

Auch unabhängig vom interdisziplinären Forschungsfeld der SynBio stellt die Recherche fachlich ausgewiesener wissenschaftlicher Expert*innen ein komplexes Problem dar. Hierzu muss zunächst definiert werden, welche Kriterien sich zur Bestimmung der wissenschaftlichen Expertise bzw. Reputation eines/r Forscher*in eignen. Dewett und Denisi (2004: 249) definieren Expertise als „the overall judgement of a scholar's standing based on their research and impact on the field as determined by experts in that field“" 3 Als Indikatoren zur Bestimmung der fachlichen Expertise bzw. Reputation werden innerhalb des Wissenschaftssystems i.d.R. bibliometrische Kennwerte herangezogen. Dazu gehören die Anzahl der Publikationen und die Zitierhäufigkeit (vgl. z. B. Shepherd, 1981: 133; Peters, 1994: 173; Weingart, 2001: 265; Dewett/Denisi, 2004: 255; Eisenegger, 2005: 69; Schimank, 2010: 234). Viele Publikationen und eine hohe Anzahl an Zitationen machen Wissenschaftler*innen öffentlich sichtbar, sodass sie als Expert*innen in ihrem Forschungsfeld wahrgenommen werden. Jedoch ist der Vergleich von mehreren Wissenschaftler*innen aufwendig, wenn einzelne Personen recherchiert

2 Die Entwicklungsarbeit am ExpertExplorer wurde durch Mittel des BMBF im Rahmen des Förderprogramms „InfectControl 2020“ gefördert (Förderkennzeichen: 03ZZ0804B).

3 "Die Gesamtbeurteilung des Ansehens einer/s Wissenschaftler*in auf der Grundlage ihrer/seiner Forschung und ihrer/seines Einflusses auf das Fachgebiet, wie es von Expert*innen auf diesem Gebiet bestimmt wird“ (eigene Übersetzung). 
und deren Publikationsanzahl und Zitierhäufigkeit erst ermittelt und dann miteinander verglichen werden sollen. An dieser Stelle kann der ExpertExplorer weiterhelfen. Im Folgenden erläutern wir die Funktionsweise des ExpertExplorers, um unsere Methode zur Recherche von Expert*innen auf dem Gebiet der SynBio transparent und nachvollziehbar zu machen.

\subsection{Funktionsweise des ExpertExplorers}

Der ExpertExplorer ${ }^{4}$ ist eine frei zugängliche Webapplikation, ${ }^{5}$ die die Suche fachlich ausgewiesener Wissenschaftler*innen insbesondere im Bereich der (bio-)medizinischen Forschung erleichtert. Die Webapplikation extrahiert aus der Datenbank Europe PubMedCentral (PMC) ${ }^{6}$ wissenschaftliche Publikationen via API-Schnittstelle. ${ }^{7}$ Die Recherche erfolgt durch die Eingabe eines zunächst breiten Suchbegriffs. Der ExpertExplorer identifiziert standardmäßig alle Publikationen, die den Suchbegriff im Titel oder in der „extensive search“ im Titel oder Abstract enthalten. Jeder Publikation ist eine Liste medizinischer Themen $(\mathrm{MeSH})^{8}$ sowie Stichwörter zugeordnet. Aus diesen Listen zieht der ExpertExplorer eine Schlagwortliste (,tags identified“), mit der die Suchanfrage weiter eingegrenzt werden kann.

Bei der Extraktion der Expert*innen liegt der Fokus auf dem/der ersten und dem/ der letzten Autor*in, wobei davon ausgegangen wird, dass diese als Hauptautor*in oder Supervisor*in fungierten (,first-last-author emphasis“). Recherchiert werden Autor*innen mit mindestens zwei Publikationen. Mit der Option ,include long tail“ werden auch Autor*innen mit nur einer Publikation extrahiert. Die Suche kann auch auf bestimmte Länder eingegrenzt werden. Das Rechercheergebnis ist eine Liste von Wissenschaftler*innen, die den zuvor gewählten Kriterien entsprechen. ${ }^{9}$

4 Frei zugänglich unter: http://webapp.expertexplorer.de/\#/ [04.12.2020].

5 Entwickelt wurde das Tool von Markus Lehmkuhl (Karlsruher Institut für Technologie) in Kooperation mit dem Science Media Center Germany, hier Volker Stollorz und Meik Bittkowski, sowie mit Yannick Milhahn (ensolorado Software und Verwaltungs UG).

$6 \quad$ PMC ist eine frei zugängliche Datenbank. Sie umfasst wissenschaftliche Publikationen aus den Bereichen Medizin, Biologie und angrenzenden Fachgebieten.

7 Als API (,application programming interface“) wird eine Programmierschnittstelle bezeichnet, die von einer Software zur Verfügung gestellt wird, damit andere Programme dort andocken und so z. B. Informationen abrufen können.

8 MeSH ist ein umfassendes, kontrolliertes Vokabular zum Indexieren von Zeitschriftenartikeln und Büchern in den Biowissenschaften.

9 Die Webapplikation wurde inhaltlich validiert, indem an einem Beispiel manuell überprüft wurde, ob die durch das Tool gelisteten Expert*innen tatsächlich inhaltlich passende Publikationen in wissenschaftlichen Fachzeitschriften aufzuweisen hatten. Zusätzlich wurde die Funktionsweise der Webapplikation durch 12 deutsche Wissenschaftsjournalist*innen getestet und evaluiert (vgl. Lehmkuhl et al., 2021). 


\subsection{ExpertExplorer: Expert*innen auf dem Gebiet der SynBio}

Ziel unseres Beitrags ist es, das Expert*innenfeld auf dem Gebiet der SynBio zu recherchieren und aufzuschlüsseln. Dies wurde unter Anwendung des ExpertExplorers erreicht. Zunächst wurde ein einschlägiger MeSH-Term für die Suche identifiziert. „Synthetic Biology" wird laut des Medical Subject Headings (MeSH) Thesaurus definiert als "field of biological research combining engineering in the formulation, design, and building (synthesis) of novel biological structures, functions, and systems. ${ }^{\text {" }}{ }^{10}$ Es ist jedoch keine Voraussetzung, einen MeSH-Term zur Suche zu verwenden; es kann jeder beliebige Suchbegriff eingesetzt werden.

Die Recherche ${ }^{11}$ erfolgte in der Suchmaske des ExpertExplores in folgenden Schritten: Abbildung 1 zeigt einen Screenshot nach Abschluss des kompletten Suchvorgangs.

Abbildung 1: Screenshot nach Abschluss des Suchvorgangs im ExpertExplorer des Science Media Center Germany (SMC)

\section{Q General Topic \\ searched 11247 valid publications \\ related to synthetic biology \\ released between 2013 and 2020 \\ and found 6037 experts \\ () Specific Topic \\ 6037 experts \\ without any tags selected \\ Found Experts}

6037 experts

from all countries

10 Synthetic Biology MeSH Descriptor Data 2020 unter: https://meshb.nlm.nih.gov/record/ ui?ui=D058615 [26.11.2020]. „Bereich der biologischen Forschung, der Methoden bei der Zusammensetzung, dem Design und dem Aufbau (Synthese) neuartiger biologischer Strukturen, Funktionen und Systeme kombiniert" (eigene Übersetzung).

11 Durchführung der Recherche am 04.01.2021. 
Auf der obersten Ebene „General Topic“ wurden folgende Begriffe bzw. Kriterien ausgewählt:

- Topic [Thema]: Synthetic Biology

- Inkl. extensive search [umfangreiche Suche]

- Publication between [Publikation zwischen]: 2013-202012

Auf der nächsten Ebene der Suchmaske „Specific Topic“ besteht die Möglichkeit, verschiedene „tags identified“ hinzuzufügen, um die Ergebnisse einzugrenzen und das jeweilige recherchierte Forschungsfeld in seiner ganzen thematischen Breite besser erfassen zu können. Dieser Schritt wurde übersprungen, da für die vorliegende Recherche die Forschungslandschaft SynBio möglichst umfassend abgedeckt werden sollte. Mit dieser Abfrage wurden 11.247 valide Publikationen geprüft und weltweit 6.037 fachlich auf dem Gebiet der SynBio ausgewiesene Wissenschaftler*innen identifiziert.

Auf der letzten und dritten Ebene „Found experts“ wurde die Liste von Expert*innen generiert. ${ }^{13}$ Gelistet wurden neben dem Namen der Wissenschaftler*innen auch deren thematischer Fokus, ihre Affiliation sowie die Länder, in denen die Forschenden aktuell arbeiten oder gearbeitet haben.

Von den weltweit im Untersuchungszeitraum identifizierten 6.037 Expert*innen ${ }^{14}$ waren zum Zeitpunkt der Recherche oder davor 488 in Deutschland tätig (8,1\%). Im internationalen Vergleich erschien das Feld der in Deutschland tätigen SynBio-Forscher*innen (im gewählten Untersuchungszeitraum) recht groß: Die USA lagen mit 2.853 Expert*innen an erster Stelle, gefolgt von China $(n=739)$ und Großbritannien $(n=578)$. An vierter Position stand bereits Deutschland ( $n=488)$; gefolgt von Japan $(n=244)$.

Insgesamt wurden von den in Deutschland tätigen Forschenden 2.969 Publikationen ${ }^{15}$ auf dem Gebiet der SynBio im Erhebungszeitraum veröffentlicht. Die durchschnittliche Anzahl an Publikationen pro Expert*in betrug 6,08 (im Vergleich weltweit: 33.496 Beiträge; durchschnittlich pro Expertin*in: 5,55). Bei einer detaillierten Analyse der Daten aus Deutschland wurde ersichtlich, dass ca. 17 \% der Expert*innen 7 oder mehr Publikationen veröffentlicht haben ( $n=83)$. Der Großteil der Wissenschaftler*in-

12 Der Erhebungszeitraum reicht von 2013 bis 2020. Im Jahr 2012 erschien eine Publikation der IAG Gentechnologiebericht zum Thema synthetische Biologie, die neben aktuellen Entwicklungen auf dem Gebiet auch deren Implikationen diskutierte (Köchy/Hümpel, 2012); nun soll daran anknüpfend die Entwicklung des Expert*innenfelds seit diesem Zeitpunkt beschrieben werden.

13 Der Abruf einer csv-Datei (Dateiformat zur Speicherung und Ausgabe von Tabellen und Listen) ist möglich.

1490 Treffer konnten aufgrund fehlender Angaben keinem Land zugeordnet werden.

15 Expert*innen mit mindestens zwei Publikationen. 
nen war an 6 oder weniger Beiträgen beteiligt: ihr prozentualer Anteil betrug ca. $83 \%$ $(n=405)$.

Die Anzahl der Zitationen dieser Publikationen von in Deutschland tätigen Forschenden betrug für den Erhebungszeitraum insgesamt 66.415. Die durchschnittliche Zitationshäufigkeit pro Publikation betrug 22,37. Hier war auffällig, dass der Anteil der Expert*innen, deren Publikationen weniger als 23 Mal zitiert wurden, lediglich ca. $20 \%$ ( $n=97)$ betrug; 80 \% der Wissenschaftler*innen wurden 23 Mal und häufiger zitiert $(n=391)$. Weltweit betrug die Zitationsanzahl 799.325, die durchschnittliche Zitierhäufigkeit lag bei 23,86 pro Publikation. An dieser Stelle zeigte sich, dass in Deutschland tätige Wissenschaftler*innen im internationalen Vergleich zwar leicht überdurchschnittlich viel publizierten, diese Publikationen aber im Durchschnitt etwas seltener zitiert wurden.

In den Tabellen 1 und 2 sind jeweils die Top Five der in Deutschland in der Vergangenheit und aktuell tätigen Expert*innen gelistet, gemessen an den Kriterien Anzahl der Publikationen und Anzahl der Zitationen. Anzumerken ist, dass ihr Anteil sowohl im Bereich der Publikationen als auch der Zitationen etwas mehr als ein Sechstel betrug. Nur ein/e Expert*in (A) ist in beiden Listen aufgeführt.

Tabelle 1: Top Five der Expert*innen (nach Anzahl der Publikationen, 2013-2020, in Deutschland tätig)

\begin{tabular}{|l|l|l|l|l|}
\hline Rang national & Rang weltweit & Expert*in *) & $\begin{array}{l}\text { Anzahl } \\
\text { Publikationen }\end{array}$ & $\begin{array}{l}\text { Anteil an Publikationen } \\
\text { gesamt, in Deutschland, } \\
\text { in Prozent }\end{array}$ \\
\hline 1 & 5 & A & 139 & 4,68 \\
\hline 2 & 9 & B & 113 & 3,81 \\
\hline 3 & 13 & C & 93 & 3,13 \\
\hline 4 & 27 & D & 65 & 2,19 \\
\hline 5 & 43 & E & 52 & 1,75 \\
\hline Rang 1-5 & & & 462 & 15,56 \\
\hline
\end{tabular}

*) Die Expert*innen sind bzw. waren nicht zwangsläufig über den gesamten Erhebungszeitraum in Deutschland tätig. 
Tabelle 2: Top Five der Expert*innen (nach Anzahl der Zitationen, 2013-2020, in Deutschland tätig)

\begin{tabular}{|l|l|l|l|l|}
\hline Rang national & Rang weltweit & Expert*in *) & Anzahl Zitationen & $\begin{array}{l}\text { Anteil an Zitationen } \\
\text { gesamt, in Deutschland, } \\
\text { in Prozent }\end{array}$ \\
\hline 1 & 21 & F & 2.503 & 3,77 \\
\hline 2 & 24 & G & 2.274 & 3,42 \\
\hline 3 & 25 & A & 2.252 & 3,39 \\
\hline 4 & 28 & H & 2.068 & 3,11 \\
\hline 5 & 39 & I & 1.890 & 2,85 \\
\hline Rang 1-5 & & & 10.987 & 16,54 \\
\hline
\end{tabular}

*) Die Expert*innen sind bzw. waren nicht zwangsläufig über den gesamten Erhebungszeitraum in Deutschland tätig.

Die Anzahl der Publikationen pro Jahr stieg von 2013 bis 2017 weltweit kontinuierlich an. So stellte sich der Bereich SynBio als wachsendes und lebendiges Forschungsfeld dar. Die Anzahl der jährlichen Publikationen von in Deutschland tätigen Wissenschaftler*innen war im Jahr 2013 mit 56 Publikationen am geringsten. In den darauffolgenden Jahren schwankte die Zahl zwischen 129 und 182 Publikationen pro Jahr (siehe Abb. 2). Der Anteil von Publikationen der in Deutschland tätigen Wissenschaftler*innen an der weltweiten Publikationszahl war im Jahr 2018 mit 10,4 \% am höchsten (2013: 4,9 \%; 2014: 8,1 \%; 2015: 9,4 \%; 2016: 6,4 \%; 2017: 8,9 \%). So leistete der Forschungsstandort Deutschland einen - wenn auch leicht schwankenden - nennenswerten Beitrag zum sich rasch entwickelnden Forschungsfeld SynBio. 
Abbildung 2: Anzahl der Publikationen auf dem Gebiet der SynBio (2013-2018)*

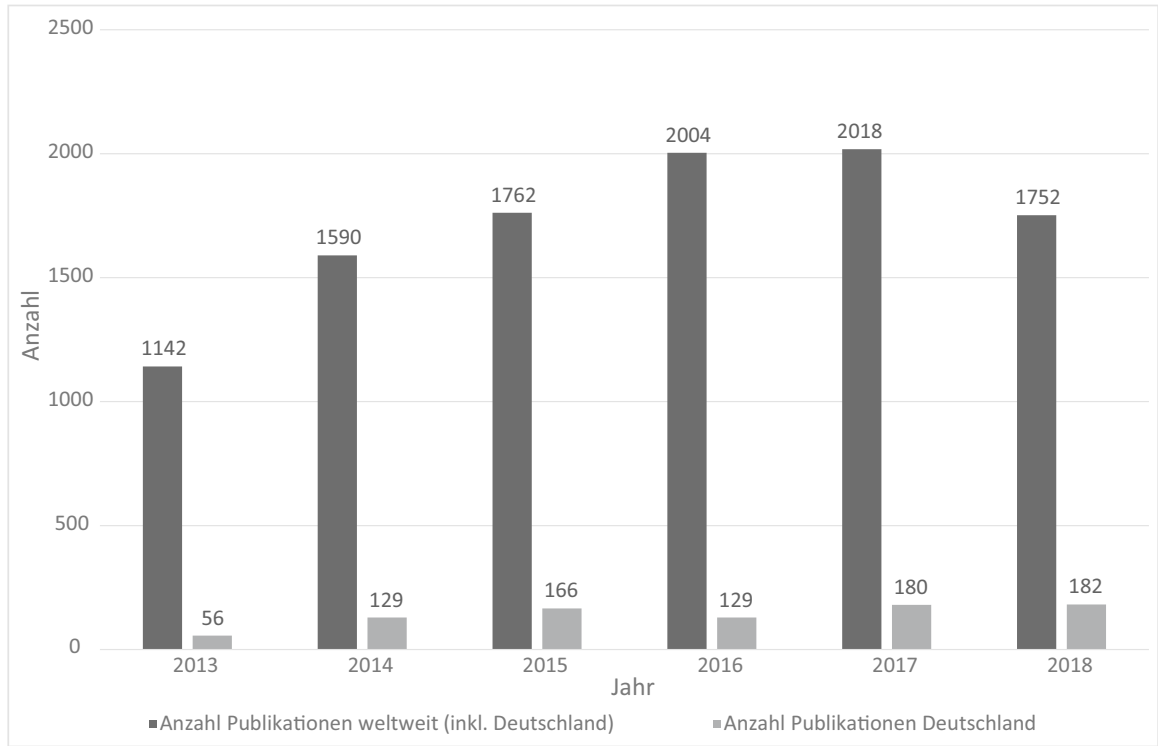

* Abgebildet wurden lediglich die Daten bis zum Jahr 2018, da einige Zeitschriften in PMC ihre Inhalte nicht direkt nach der Veröffentlichung zur Verfügung stellen, sondern erst mit Verzögerung. Zudem wurden im Laufe des Untersuchungszeitraums Zeitschriften sowohl neu hinzugefügt als herausgenommen: somit können themenrelevante Zeitschriften möglicherweise nicht über den ganzen Zeitraum verfügbar gewesen sein.

\subsection{Fazit und Reflexion}

Ein möglicher Indikator zur Identifikation fachlich einschlägiger Wissenschaftler*innen ist, wie oben ausgeführt, die wissenschaftliche Reputation. Als ein Kriterium hierfür eignet sich unter anderem die Publikations- und Zitierhäufigkeit eines/einer Wissenschaftler*in. An dieser Stelle knüpft der ExpertExplorer an. Die Webapplikation bezieht die relevanten Informationen aus der Datenbank PMC: Dies ermöglicht eine effiziente Suche medizinischer und naturwissenschaftlicher Publikationen weltweit. So ist die Anwendung gut geeignet, um die relevanten und aktuell Forschenden im Bereich der Medizin und Naturwissenschaften zu identifizieren. Einschränkend erwähnt werden sollte jedoch, dass gerade auf dem heterogenen Gebiet der SynBio auch (interdisziplinäre) geistes- und sozialwissenschaftliche Aspekte eine wichtige Rolle spielen. Die Veröffentlichungen aus diesen Disziplinen werden von der Datenbank PMC nur begrenzt erfasst. Somit liegt der Fokus bei der Expert*innenauswahl auf der naturwissenschaftlichen und medizinischen Forschung. Um Expert*innen aus anderen Bereichen zu finden, ist eine zusätzliche Suche in den jeweiligen Fachdatenbanken oder 
bspw. auch in einer Datenbank wie Web of Science notwendig, die mit verschiedenen Indizes (Social Sciences Citation, Arts \& Humanities Citation) auch die Gebiete Kunst sowie Sozial- und Geisteswissenschaften abdecken.

Des Weiteren ist anzumerken, dass englischsprachige Veröffentlichungen im Vordergrund stehen. Anderssprachige Publikationen werden nur eingeschränkt bei der Suche nach den thematisch relevanten Forschenden berücksichtigt. Auch Rankings der Expert*innen und die Vergleiche der Zitationshäufigkeiten und Publikationszahlen haben daher eine begrenzte Aussagekraft.

Zur konkreten Anwendung des ExpertExplorers ist zu sagen, dass der Einsatz der booleschen Operatoren AND und OR die Suche und Einschränkung der Publikationen resp. Expert*innen auf der mittleren Ebene der Suchmaske erleichtert. Durch die Kombination verschiedener „tags identified“ lassen sich die Suchergebnisse thematisch eingrenzen oder erweitern. Mit der Auswahl der Operatoren und Begriffe findet aber auch immer eine subjektive Auswahl durch den/die jeweilige Nutzer*in statt. So sind - je nach Fachkenntnis des/der Nutzer*in und der damit verbundenen Auswahl der tags unterschiedliche Ergebnisse möglich, die eventuell auch das zu eruierende Themenfeld nicht vollständig abdecken können. Für eine erfolgreiche und effiziente Recherche im ExpertExplorer ist, wie gerade erwähnt, eine gewisse Fachkenntnis vonnöten, um die geeigneten und relevanten Suchbegriffe zu kennen - aber dies ist bei jeder Form der Recherche der Fall.

Unsere Analyse unter Anwendung des ExpertExplorers zeigt, dass der Forschungsstandort Deutschland zur Entwicklung des stetig wachsenden Forschungsgebiets der SynBio im internationalen Vergleich einen stabilen und fundierten Beitrag leistet. So ist die Anzahl der in Deutschland tätigen Expert*innen über den gesamten Erhebungszeitraum im Vergleich zur Anzahl der Expert*innen weltweit $(\mathrm{n}=6.037)$ relativ groß ( $n=488$; Anteil: 8,1 \%). Gemessen an der Anzahl der weltweit identifizierten Wissenschaftler*innen rangiert Deutschland - hinter den USA, China und Großbritannien - auf Platz 4. Auch wenn im Jahr 2013 im Vergleich zu den darauffolgenden Jahren weniger Publikationen zu verzeichnen sind, liegt der Anteil von Publikationen der in Deutschland tätigen Wissenschaftler*innen an der weltweiten Publikationszahl gemessen zwischen 6,4 \% im Jahr 2016 und 10,4 \% im Jahr 2018. Des Weiteren ist ein/e Expert*in (A) am Forschungsstandort Deutschland hervorzuheben, der/die konstante Forschungsleistungen erbringt und im internationalen Vergleich sowohl hinsichtlich der Publikationsanzahl als auch der Zitierhäufigkeit im oberen Bereich der internationalen Expert*innenliste zu finden ist.

Abschließend ist zu sagen, dass die Webapplikation ExpertExplorer sowohl für (Wissenschafts-)Journalist*innen als auch für die fachlich interessierte Öffentlichkeit eine 
gute Möglichkeit bietet, um schnell und umfassend einen aktuellen Überblick über fachlich ausgewiesene Expert*innen auf den Feldern der biomedizinischen Forschung zu erhalten. Darüber hinaus lassen sich mithilfe der Erhebung bibliometrischer Kennwerte wie Publikationsanzahl und Zitierhäufigkeit neben dem Status quo auch Entwicklungen von Forschungslandschaften hinsichtlich wissenschaftlicher Expertise resp. Reputation auch über längere Zeiträume beschreiben und vergleichen.

\subsection{Literaturverzeichnis}

Dewett, T./Denisi, A. S. (2004): Exploring scholarly reputation: It's more than just productivity. In: Scientometrics 60: 249-272.

Eisenegger, M. (2005): Reputation in der Mediengesellschaft. VS, Wiesbaden.

GASB = German Association for Synthetic Biology (2020): What is synthetic biology? Unter: https:// www.synthetischebiologie.org/what-is-synthetic-biology [27.01.2021].

Köchy, K./Hümpel, A. (Hrsg.) (2012): Synthetische Biologie. Entwicklung einer neuen Ingenieurbiologie? Themenband der Interdisziplinären Arbeitsgruppe Gentechnologiebericht. Forum W, Dornburg.

Köhler, N. et al. (2017): Synthetische Biologie. In: Journal of Consumer Protection and Food Safety 12: 61-65. DOI: 10.1007/s00003-016-1068-7.

Lehmkuhl, M. et al. (2021): ExpertExplorer: Ein praktisches Tool für die Integration wissenschaftlicher Expert(inn)en in zivilgesellschaftliche Debatten. Vortrag auf der 5. Jahrestagung der Fachgruppe Wissenschaftskommunikation der DGPuK vom 3. bis 5. Februar 2021 in Göttingen.

Peters, H. P. (1994): Wissenschaftliche Experten in der öffentlichen Kommunikation über Technik, Umwelt und Risiken. In: Neidhardt, F. (Hrsg.): Öffentlichkeit, öffentliche Meinung, soziale Bewegungen. Westdeutscher Verlag, Opladen: 169-190.

Schimank, U. (2010): Reputation statt Wahrheit: Verdrängt der Nebencode den Code? In: Soziale Systeme 16: 233-242.

Shepherd, R. G. (1981): Selectivity of sources: reporting the marijuana controversy. In: Journal of Communication 31: 129-137.

Weingart, P. (2001): Die Stunde der Wahrheit? Zum Verhältnis der Wissenschaft zu Politik, Wirtschaft und Medien in der Wissensgesellschaft. Velbrück Wissenschaft, Weilerswist. 